\title{
Immune biomarker correlates from a Phase II study of ipilimumab (IPI) with carboplatin and paclitaxel (CP) in patients with unresectable stage III or IV metastatic melanoma (MM)
}

Réjean Lapointe ${ }^{1 *}$, Rahima Jamal ${ }^{2}$, Leon van Kempen ${ }^{3}$, Pamela Thebault ${ }^{4}$, Karl Belanger ${ }^{2}$, Jennifer Friedmann ${ }^{5}$, Jean-Pierre Ayoub², Eftihia Cocolakis ${ }^{3}$, Shirin Kazemi ${ }^{3}$, Jeanne Dionne ${ }^{2}$, Caroline Lambert ${ }^{3}$, Huy Le ${ }^{3}$, Cecile Grange ${ }^{6}$, Jean-Francois Cailhier ${ }^{6}$, Alan Spatz ${ }^{3}$, Wilson Miller ${ }^{3}$

From 30th Annual Meeting and Associated Programs of the Society for Immunotherapy of Cancer (SITC 2015) National Harbor, MD, USA. 4-8 November 2015

\section{Background}

Pivotal studies with anti-CTLA-4 (Ipilimumab or IPI) demonstrated increased overall survival (OS) both as single agent (10.1 months) and with DTIC (11.2 months). We've previously reported an encouraging safety profile with IPI plus CP and early efficacy results. We now report updated 1-year OS and immune biomarker correlates of patient response.

\section{Methods}

30 patients were randomized in a 1:2 ratio to arm A (C $(\mathrm{AUC}=6)$ and $\mathrm{P}(175 \mathrm{mg} / \mathrm{m} 2)$ every 3 weeks $\times 5$ and IPI $(3 \mathrm{mg} / \mathrm{kg})$ every 3 weeks $\times 4$ starting at week 4$)$ or arm B (similar dosing to arm A except IPI was given on week after $\mathrm{CP}$ ). Tumor biopsies were collected at screening and week 8 , and immune monitoring bloods were collected throughout.

\section{Results}

Median OS was 16.1 months, with a 1-year OS of 56.5\% for all patients with no differences between arms. Overall median follow-up was 23.2 months. Best overall response rate (BORR) and disease control rate (DCR) were $26.7 \%$ and $56.7 \%$ by irRC. BORR in patients whose tumors were wild type for BRAF and NRAS was $44 \%$, compared to $8 \%$ in patients with a mutation in BRAF or NRAS. Clinical responses correlated with the abundance of peri and intratumoral CD3+ inflammatory cells in the pretreatment biopsy, but not with $\mathrm{CD} 4 / \mathrm{CD} 8$ ratios or CD20 infiltrate. Circulating levels of some chemokines were elevated in non-responders compared to responders. While IPI influenced B cells and monocyte differentiation, this did not correlate with clinical outcome. Lower levels of PD-1 on CD4 and CD8 T cells were observed in responders compared to non-responders.

\section{Authors' details}

'Université de Montréal / Centre de Recherche du Centre Hospitalier de I'Université de Montreal (CRCHUM), Montreal, PQ, Canada. ${ }^{2}$ Centre Hospitalier de Université de Montréal, Montreal, PQ, Canada. ${ }^{3}$ Jewish General Hospital, McGill University, Montreal, PQ, Canada. ${ }^{4}$ Université de Montréal, Montreal, $P Q$, Canada. ${ }^{5}$ Jewish General Hospital, McGill University, Montreal, PQ, Canada. ${ }^{6}$ Université de Montréal, Montreal, PQ, Canada.

Published: 4 November 2015

\section{doi:10.1186/2051-1426-3-S2-P259}

Cite this article as: Lapointe et al:: Immune biomarker correlates from a Phase II study of ipilimumab (IPI) with carboplatin and paclitaxel (CP) in patients with unresectable stage III or IV metastatic melanoma (MM). Journal for ImmunoTherapy of Cancer 2015 3(Suppl 2):P259. 